\title{
Autofocals: Evaluating Gaze-Contingent Eyeglasses for Presbyopes
}

\author{
Nitish Padmanaban \\ Stanford University \\ nit@stanford.edu
}

\author{
Robert K. Konrad \\ Stanford University \\ rkkonrad@stanford.edu
}

\author{
Gordon Wetzstein \\ Stanford University \\ gordon.wetzstein@stanford.edu
}

\begin{abstract}
Presbyopia, the loss of accommodation due to the stiffening of the crystalline lens in the eye, affects nearly $20 \%$ of the population worldwide. Traditional forms of presbyopia correction use fixed focal elements that inherently trade off field of view or stereo vision for a greater range of distances at which the wearer can see clearly. However, none of these offer the same natural refocusing enjoyed in youth. In this work, we built a new type of presbyopia correction, dubbed "autofocal," which externally mimics the natural accommodation response of the eye by combining data from eye trackers and a depth sensor, and then automatically drives focus-tunable lenses. We evaluated autofocals against progressives and monovision in a user study; compared to these traditional corrections, autofocals maintain better visual acuity at all tested distances, allow for faster and more accurate visual task performance, and are easier to refocus with for a majority of wearers.
\end{abstract}

\section{CCS CONCEPTS}

- Human-centered computing $\rightarrow$ User studies; Accessibility technologies; $\bullet$ Hardware $\rightarrow$ Sensor applications and deployments.

\section{KEYWORDS}

presbyopia, computational optometry

\section{ACM Reference Format:}

Nitish Padmanaban, Robert K. Konrad, and Gordon Wetzstein. 2019. Autofocals: Evaluating Gaze-Contingent Eyeglasses for Presbyopes. In Proceedings of SIGGRAPH '19 Talks. ACM, New York, NY, USA, 2 pages. https: //doi.org/10.1145/3306307.3328147

\section{INTRODUCTION}

Vision is one of the most, if not the most, important senses that humans use to navigate their everyday worlds. Unfortunately, as people age, their ability to accommodate, or refocus the crystalline lenses in their eyes to near distances, steadily decreases until most cannot view anything within arm's reach in sharp focus [Duane 1912]. This loss in range of clear vision is known as presbyopia and affects over a billion people worldwide [Holden et al. 2008].

Traditional presbyopia corrections use fixed-focus optics. Some typical corrections used today include reading glasses, bifocals, progressive addition lenses, and monovision, but these all fall short of restoring the quality of vision they once enjoyed in their youth. Reading glasses, besides the inconvenience, can also be forgotten or misplaced. Bifocals, trifocals, and progressives restrict the field of view at any given desired focal distance and introduce distortions in

Permission to make digital or hard copies of part or all of this work for personal or classroom use is granted without fee provided that copies are not made or distributed for profit or commercial advantage and that copies bear this notice and the full citation on the first page. Copyrights for third-party components of this work must be honored. For all other uses, contact the owner/author(s).

SIGGRAPH '19 Talks, July 28 - August 01, 2019, Los Angeles, CA, USA

(c) 2019 Copyright held by the owner/author(s).

ACM ISBN 978-1-4503-6317-4/19/07.

https://doi.org/10.1145/3306307.3328147

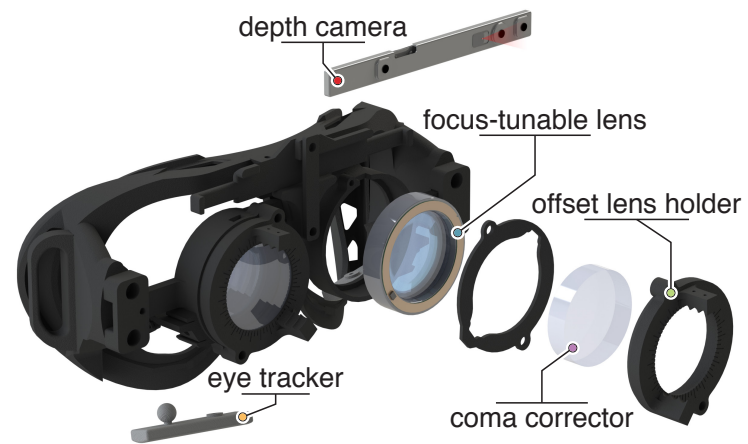

Figure 1: The prototype, with focus-tunable Optotune EL30-45 lenses, RealSense R200 depth sensor, Pupil Labs eye trackers, and offset lenses on a universal optometrist trial lens frame. Offset lenses enable support of a wider range of visual aberrations. Adapted from Padmanaban et al. [2019].

regions where the refractive power transitions. As for monovision and the various simultaneous vision approaches, studies consistently show that they too fall short of reading glasses for visual acuity or stereoacuity, with some prospective wearers rejecting them entirely due to poor or unnatural vision [Back et al. 1992; Erickson and Schor 1990; Harris et al. 1992; Sheedy et al. 1991, 1988].

Traditional methods of correction use fixed focal elements to approximate vision that was once achieved by varifocal lenses in the wearer's eyes, but varifocal elements such as liquid-filled membrane lenses or liquid-crystal (LC) lenses suggest a new approach. For example, there have been proposals using liquid lenses in eyeglasses-based correction [Hasan et al. 2017a]. LC lenses may be even more flexible in that they can directly address phase across the field of view, allowing for more varied corrections with a single element, with much recent work on improving focusing time and range [Chen et al. 2014; Lin and Chen 2013; Lin et al. 2014].

\section{EVALUATING AUTOFOCALS}

Lenses such as the above have been used in prototypes of electronically tunable eyewear, controllable via either manual input or a depth sensor [Hasan et al. 2017b; Li et al. 2006; Wang et al. 2014]. None of these solutions emulate natural accommodation: looking around and having focus simply work. Implementing this ideal would require both a depth camera and robust, affordable eye trackers. For example, despite having a depth sensor, Hasan et al. [2017b] omit eye tracking and therefore force the user to focus to the object directly in the center of the user's vision. Drawing inspiration from gaze-contigent varifocal systems for virtual reality [Konrad et al. 2016; Padmanaban et al. 2017], we present autofocals (Figure 1), which we believe to be the first focus-tunable eyewear for presbyopia correction to incorporate eye trackers and a depth camera. With this system, we compare our performance against that of commonly prescribed treatments, specifically progressives and monovision. 

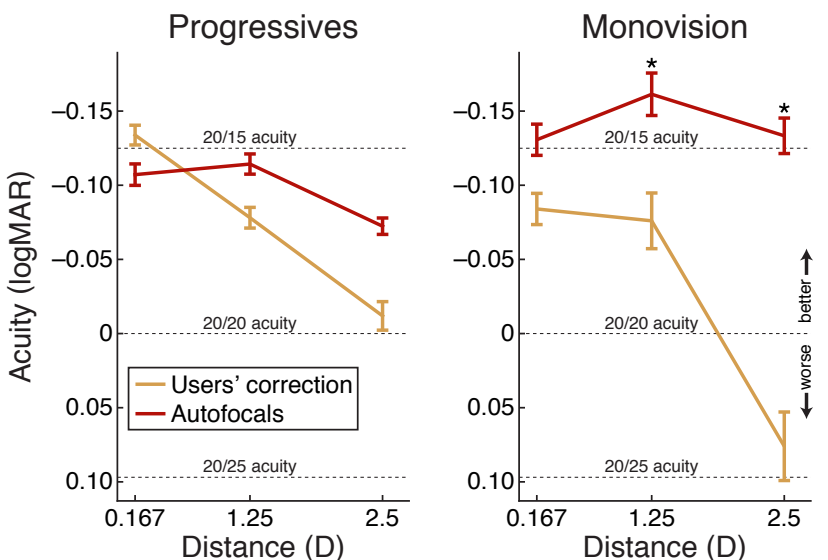

Figure 2: Acuity measurements comparing presbyopes wearing progressives or monovision to autofocals. Autofocals are better at most distances, and comparable to progressives at the far distance. Stars indicate $p<0.05$; error bars are SE. Adapted from Padmanaban et al. [2019].

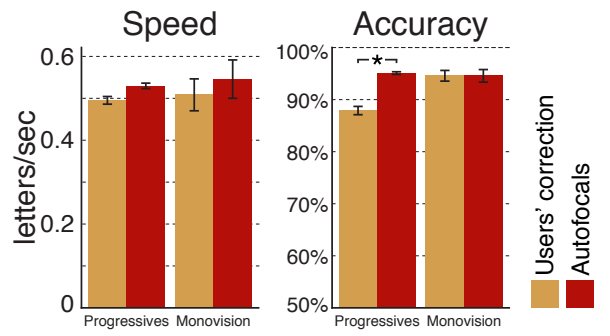

Figure 3: Speed and accuracy of a letter matching task. Autofocals are faster than traditional correction and significantly more accurate than progressives $(p<0.05)$. Error bars are SE. Adapted from Padmanaban et al. [2019].

We evaluated autofocals on a total of 56 users across a set of visual, task performance, and qualitative metrics: visual acuity, contrast sensitivity, letter matching, and preference. For the first study ( $n=19$, ages $52-70$ ), we observed that autofocals maintain visual acuity of about $0.1 \log$ MAR better than $20 / 20$ across all tested distances (Figure 2). This presents a contrast with both monovision and progressive lenses, which exhibit a downward trend in acuity at nearer distances, resulting in autofocals outperforming both at nearly all distances. No significant difference was observed with contrast sensitivity, which serves to verify that focus-tunable lenses do not have any unforeseen effects on visual contrast.

In the task performance study ( $n=18$, ages $52-70)$, we asked users to perform a letter matching task (letter size 0.1 logMAR). We found that autofocals allowed progressives wearers to perform significantly more accurately, while also going faster (Figure 3). The difference was less pronounced for monovision wearers, but they still performed on average faster while wearing autofocals. These results can be attributed to the fact that moving one's head to adjust focus is slower. Monovision wearers do not require head movements, but may slow down due to reduced acuity.

Finally, in the preference study ( $n=37$, ages $50-66$ ), we asked users to compare autofocals against their own correction. While our current autofocal prototype was rated as less comfortable than their own eyeglasses, a clear majority rated autofocals as being easier to refocus [Padmanaban et al. 2019]. Furthermore, we compared autofocals against a version that uses only a depth tracker as in Hasan et al. [2017b], and found that people prefer eye-tracked focus estimation by a significant margin.

Complete results can be found in Padmanaban et al. [2019].

\section{CONCLUSION}

Presbyopia, despite being a nearly universal problem in old age, still does not have an ideal commercially available solution. We built a prototype autofocal system for evaluating the utility and current state of focus-tunable lens technology for correcting presbyopia. Even with an early prototype, autofocals often outperform traditional forms of correction, were preferred over presbyopes' own daily correction as being easier to refocus and a more convenient technology, and improve presbyopes' ability to switch tasks or between multiple working distances. Thus, autofocals have important ramifications for real-world tasks, a promising result for continued pursuit of focus-tunable presbyopia corrections.

\section{ACKNOWLEDGMENTS}

This project was supported by Intel, a Sloan Fellowship, the Okawa Foundation, the National Science Foundation (NSF; 1553333, 1839974), an NSF Graduate Research Fellowship, and an NVIDIA Graduate Fellowship.

\section{REFERENCES}

Arthur Back, Timothy Grant, and Narelle Hine. 1992. Comparative visual performance of three presbyopic contact lens corrections. Optom. Vis. Sci. 69, 6 (1992), 474-480.

Hung-Shan Chen, Ming-Syuan Chen, and Yi-Hsin Lin. 2014. Electrically Tunable Ophthalmic Lenses for Myopia and Presbyopia Using Liquid Crystals. Mol Cryst Liq Cryst 596, 1 (2014), 88-96.

Alexander Duane. 1912. Normal values of the accommodation at all ages. fournal of the American Medical Association 59, 12 (1912), 1010-1013.

Paul Erickson and Clifton Schor. 1990. Visual function with presbyopic contact lens correction. Optom. Vis. Sci. 67, 1 (1990), 22-28.

Michael G Harris, James E Sheedy, and Cheslyn M Gan. 1992. Vision and task performance with monovision and diffractive bifocal contact lenses. Optom. Vis. Sci. 69, 8 (1992), 609-614.

Nazmul Hasan, Aishwaryadev Banerjee, Hanseup Kim, and Carlos H Mastrangelo. 2017a. Tunable-focus lens for adaptive eyeglasses. Opt. Express 25, 2 (2017).

Nazmul Hasan, Mohit Karkhanis, Fariha Khan, Tridib Ghosh, Hanseup Kim, and Carlos H Mastrangelo. 2017b. Adaptive Optics for Autofocusing Eyeglasses. In Applied Industrial Optics: Spectroscopy, Imaging and Metrology. OSA, AM3A-1.

Brien A Holden, Timothy R Fricke, S May Ho, Reg Wong, Gerhard Schlenther, Sonja Cronjé, Anthea Burnett, Eric Papas, Kovin S Naidoo, and Kevin D Frick. 2008. Global vision impairment due to uncorrected presbyopia. Arch Ophthalmol 126, 12 (2008).

Robert Konrad, Emily A. Cooper, and Gordon Wetzstein. 2016. Novel optical configurations for virtual reality: Evaluating user preference and performance with focus-tunable and monovision near-eye displays. In Proc. SIGCHI.

Guoqiang Li, David L Mathine, Pouria Valley, Pekka Äyräs, Joshua N Haddock, MS Giridhar, Gregory Williby, Jim Schwiegerling, Gerald R Meredith, Bernard Kippelen, et al. 2006. Switchable electro-optic diffractive lens with high efficiency for ophthalmic applications. PNAS 103, 16 (2006), 6100-6104.

Yi-Hsin Lin and Hung-Shan Chen. 2013. Electrically tunable-focusing and polarizerfree liquid crystal lenses for ophthalmic applications. Opt. Express 21, 8 (2013).

Yi-Hsin Lin, Hung-Shan Chen, and Ming-Syuan Chen. 2014. Electrically Tunable Liquid Crystal Lenses and Applications. Mol Cryst Liq Cryst 596, 1 (2014), 12-21.

Nitish Padmanaban, Robert Konrad, Tal Stramer, Emily A. Cooper, and Gordon Wetzstein. 2017. Optimizing virtual reality for all users through gaze-contingent and adaptive focus displays. PNAS 114 (2017), 2183-2188. Issue 9.

Nitish Padmanaban, Robert Konrad, and Gordon Wetzstein. 2019. Autofocals: Evaluating Gaze-Contingent Eyeglasses for Presbyopes. Science Advances (2019).

James E Sheedy, Michael G Harris, Matthew R Bronge, Sharon M Joe, and Melanie A Mook. 1991. Task and visual performance with concentric bifocal contact lenses. Optom. Vis. Sci. 68, 7 (1991), 537-541.

James E Sheedy, Michael G Harris, Leslie Busby, Eileen Chan, and Irene Koga. 1988. Monovision contact lens wear and occupational task performance. Optom. Vis. Sci. 65, 1 (1988), 14-18.

Lihui Wang, Alvaro Cassinelli, Hiromasa Oku, and Masatoshi Ishikawa. 2014. A pair of diopter-adjustable eyeglasses for presbyopia correction. In Proc. SPIE, Vol. 9193. 